\title{
Polarization properties of self-diffraction in sillenite crystals: reflection volume gratings
}

\author{
S. Mallick \\ Institut d'Optique, Centre Universitaire, Bǎtiment 503, B.P. 147, 91403 Orsay Cedex, France
}

M. Miteva and L. Nikolova

Central Laboratory for Optical Storage and Processing of Information, P.O. Box 95, Sofia 1113, Bulgaria

Received July 8, 1996

\begin{abstract}
We study two-beam interaction in photorefractive sillenite crystals in reflection geometry and derive analytic expressions for the signal gain and its polarization direction in the presence of a strong pump beam. The crystal is cut normal to one of the crystallographic axes, and no external electric field is applied. Crystal absorption and its natural optical activity are taken into account. The coupling effects are strongly dependent on the polarization directions of the interacting beams. We determine the optimal polarization directions and optimal crystal thickness that give maximum signal gain. Experimental results are in excellent agreement with theoretical calculations. (C) 1997 Optical Society of America [S0740-3224(97)01505-1]
\end{abstract}

\section{INTRODUCTION}

The phenomenon of two-beam coupling in photorefractive materials has been extensively studied, and a large number of applications in image processing, optical interconnection, and optical computing have been reported. ${ }^{1}$ The two light beams interfere and create a refractive-index grating in the material; then, they are self-diffracted from the grating they create. The processes of writing and reading thus take place simultaneously. The refractiveindex grating and the interference-fringe pattern are in general spatially shifted with respect to each other, and this results in an unsymmetrical exchange of energy between the interacting beams. A majority of research papers treating two-beam interaction have considered the codirectional geometry wherein the two beams propagate practically parallel to each other, generating a transmission grating. The case of counterpropagating beams in which a reflection grating mediates the exchange of energy has attracted less attention. Two-beam interaction in this geometry has been analyzed by $\mathrm{Yeh}^{2}$ for a $\mathrm{BaTiO}_{3}$ crystal, by Ewbank et al. ${ }^{3}$ for a Rh-doped strontium barium niobate crystal, and by Erbschloe et $a l .{ }^{4}$ for a $\mathrm{LINbO}_{3}$ crystal. An important application of the contradirectional two-beam coupling is the generation of phaseconjugate waves by stimulated photorefractive backscattering. ${ }^{5-7}$

A very interesting class of photorefractive media is that of sillenite crystals such as bismuth silicon oxide $\left(\mathrm{Bi}_{12} \mathrm{SiO}_{20}\right.$ or $\left.\mathrm{BSO}\right)$, bismuth germanium oxide $\left(\mathrm{Bi}_{12} \mathrm{GeO}_{20}\right.$ or $\mathrm{BGO}$ ) and bismuth titanium oxide $\left(\mathrm{Bi}_{12} \mathrm{TiO}_{20}\right.$ or BTO). These materials have a faster response time as compared with $\mathrm{BaTiO}_{3}, \mathrm{LiNbO}_{3}$, or SBN, but they show lower gain owing to the low value of their electro-optic coefficient. The sillenite crystals are opti- cally active and become, in addition, linearly birefringent in the presence of an electric field. Owing to the concomitant presence of optical activity and linear birefringence, light diffraction in these crystals exhibits complex polarization effects. A large number of authors ${ }^{8-11}$ have studied the polarization characteristics of light diffracted by a static, prewritten transmission grating as a function of the polarization direction of the readout beam. It was demonstrated that under certain conditions the emerging readout beam and the image-bearing diffracted beam have orthogonal polarizations, and consequently the signal-to-noise ratio in the output image can be greatly enhanced when the strong readout beam is eliminated with a linear polarizer. Polarization effects in two-beam coupling in the presence of self-diffraction have also been widely studied. However, as in high-gain materials, most of the papers on sillenite crystals deal with the codirectional geometry. ${ }^{12,13} \mathrm{Ja}^{14,15}$ was the first to analyze the contradirectional two-beam coupling in BGO and to measure signal gain as a function of the incident angle of the beams and of the initial beam intensity ratio. Ja, however, neglected the optical activity and used numerical methods to determine the transmission coefficients of the beams; analytic results were obtained only when crystal absorption was neglected. Kukhtarev et al. ${ }^{16}$ derived coupled-wave equations for the two-beam interaction in reflection geometry, taking into account both optical activity and linear birefringence. They solved the coupledwave equations by an iterative method and predicted that self-diffraction might induce a polarization rotation of a few degrees per centimeter. In a recent paper ${ }^{17}$ two of us studied the self-diffraction effects in sillenite crystals between a forward-propagating beam and its backreflected beam. We found that the light beam exiting a 4-mm 
thick BTO crystal varied in intensity by as much as $\pm 40 \%$ when the polarization direction of the incident beam was changed.

In this paper we derive analytic expressions for the amplification of a weak signal beam and its polarization state in a reflection geometry as a function of the polarization directions of the signal and pump beams, optical activity, refractive-index modulation, and crystal thickness. Crystal absorption is taken into account, but the pump depletion due to energy transfer is neglected. The crystal wafer is cut normal to one of the crystallographic axes [Fig. 1(a)]. The space-charge field that is due to the reflection grating is parallel to this axis $[\langle 001\rangle$ in Fig. 1(a)]. Due to the Pockels effect, the cubic sillenite crystal becomes linearly birefringent; the birefringent axes, called $x$, and $y$ in the figure, lie in the plane of the wafer and are at $45^{\circ}$ with respect to the crystallographic axes $\langle 100\rangle$ and $\langle 010\rangle$. The refractive index for a light beam propagating along the $z$ axis is $n_{0}+(1 / 2) n_{0}{ }^{3} r E_{\mathrm{sc}}$ or $n_{0}$ - $(1 / 2) n_{0}{ }^{3} r E_{\mathrm{sc}}$ according to whether the electric vector of the light beam is along the $x$ axis or along the $y$ axis; here $n_{0}$ is the refractive index of the cubic crystal and $r$ the electro-optic coefficient. If the crystal is cut normal to the $\langle 111\rangle$ crystallographic axis, the refractive index for light propagating along this axis is independent of its polarization direction ${ }^{18}$ and its value is $n_{0}$ $+(1 / 2 \sqrt{ } 3) n_{0}{ }^{3} r E_{\mathrm{sc}}$. In this configuration, the polarization effects are less important and are easy to explain; however, the signal gain is much lower due to the smaller value of the refractive-index modulation.

No bias electric field is applied to the crystal. The displacement of the free-charge carriers is by diffusion alone, and thus the refractive-index grating is shifted through a quarter of its period with respect to the interference fringes. The direction of the shift is taken to be such that the pump beam self-diffracted from the grating adds in phase to the signal when the pump-beam polarization is along the $x$ axis and in antiphase when the pump-beam polarization is along $y$ axis.

In the next section we calculate the fringe contrast as a function of $z$, and the $x$ and $y$ amplitude components of

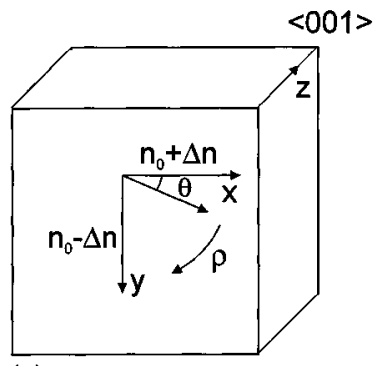

(a)

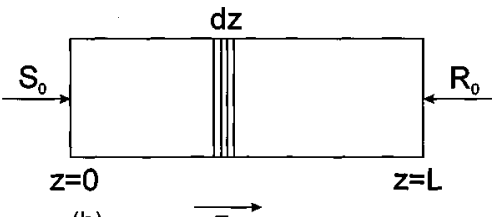

(b) $\mathbf{z}^{\rightarrow}$

Fig. 1. Crystal orientation and geometry for contradirectional two-wave mixing. The pump beam $R_{0}$ makes a small angle with the $z$ axis such that the light reflected at the external face $z$ $=L$ does not mix with the outcoming signal. $\Delta n=n_{0}{ }^{3} r E_{\mathrm{sc}} / 2$. the amplified signal at the exit plane. In general, numerical integration has to be carried out to yield the final result. Analytic expressions for the signal gain and its polarization direction are, however, obtained in Appendix A for the case in which $\pi \Delta n_{\max } / \lambda \rho<1$; here $\Delta n_{\max }$ is the saturation value of the refractive-index modulation obtained with fringes of unit contrast, and $\rho$ is the angle of rotation of the light vector per unit thickness of the crystal. This condition is amply satisfied by BSO and BGO crystals. In Section 3 we compare the analytic results with the exact numerically calculated values and with the experimentally measured data. Some concluding remarks about the optimal beam polarizations and crystal thickness are given in the last section.

\section{TWO-BEAM COUPLING IN REFLECTION GEOMETRY IN THE PRESENCE OF OPTICAL ACTIVITY}

The geometry used to calculate the signal gain is shown in Fig. 1(b). The weak signal $S$ and the strong pump $R$ are propagating in opposite directions practically parallel to the $z$ axis, and the polarization vectors lie in the $x-y$ plane. The crystal is assumed to have antireflection coatings. The eigenwaves for a crystal having both circular and linear birefringence are elliptic. However, in our case, because no bias electric field is applied to the crystal and because the space-charge field generated by the low-contrast fringes is only a few hundred volts per centimeter, the linear birefringence is very small compared with the circular birefringence. Under this condition it is legitimate to suppose that the propagation of light beams inside the crystal is dictated by optical activity only; the linear birefringence results in diffraction of light. The validity of this assumption is amply justified by the excellent agreement between theoretical and experimental results. All angles are measured with respect to the $x$ axis in the clockwise sense for an observer situated to the left of the crystal [see Fig. 1(b)] and looking in the positive $z$ direction (the pump beam $\mathrm{R}$ is entering his eyes). The polarization vector of the signal beam makes an angle $\theta$ with the $x$ axis at the entrance face of the crystal $(z=0)$; at depth $z$ it makes an angle $\theta$ $+\rho z$. The electric vector of the pump as it enters the crystal at $z=L$ is chosen to make an angle $\theta+\rho L$ with the $x$ axis. The polarization vector of the pump beam as it progresses in the negative $z$ direction rotates in the anticlockwise sense. With this disposition the electric vectors of the signal beam (without amplification) and the pump beam remain parallel to each other throughout the crystal, and the initial fringe contrast at $z=0$ is optimal. Owing to the exchange of energy between the beams, the fringe contrast $m$ becomes a function of $z$. To start with we determine this dependence, because the diffraction efficiency varies with $m$.

\section{A. Variation of Fringe Contrast $\boldsymbol{m}$ as a Function of $z$}

Since the pump is much stronger than the signal, the interference fringes are of low contrast, and under this condition the modulation of the crystal refractive index can be written $\Delta n=m \Delta n_{\max } ; \Delta n_{\max }$ depends on the nature of the crystal and the geometry of the experimental setup. 
The amplitude diffracted by a thin phase grating of thickness $\mathrm{d} z$ is $R(\pi / \lambda) m \Delta n_{\max } \mathrm{d} z, R$ being the amplitude of the readout beam; the factor $\exp (j \pi / 2)$ that appears in the expression for the amplitude diffracted by a phase grating is eliminated because the phase grating is shifted through a quarter of a period. The fringe contrast at a depth $z$ is

$$
m(z)=2 S_{\|} R /\left(R^{2}+S^{2}\right) \approx 2 S_{\|} / R,
$$

where $S_{\|}$is the component of the signal amplitude (initial signal plus the contribution of the diffraction process) that is polarized parallel to $R$. The amplitude $S_{\|}$varies with $z$ owing to the diffraction process and also owing to crystal absorption. The pump-beam amplitude variations are due to crystal absorption alone. Thus

$$
\mathrm{d} m=2 \mathrm{~d} S_{\|} / R-2 S_{\|} \mathrm{d} R / R^{2} .
$$

We first calculate the contribution to $S_{\|}$from the pump beam diffracted at the elementary grating situated at $z$. The $x, y$ components of the pump amplitude at $z$ are

$$
\begin{aligned}
& R_{x}=R \cos (\theta+\rho z), \\
& R_{y}=R \sin (\theta+\rho z) .
\end{aligned}
$$

The $x, y$ components of the amplitude diffracted by the grating of thickness $\mathrm{d} z$ situated at $z$ are

$$
\begin{aligned}
\mathrm{d} U_{x} & =R \cos (\theta+\rho z)(\pi / \lambda) m \Delta n_{\max } \mathrm{d} z \\
& =2 S_{\|} \beta \cos (\theta+\rho z) \mathrm{d} z \\
\mathrm{~d} U_{y} & =-2 S_{\|} \beta \sin (\theta+\rho z) \mathrm{d} z
\end{aligned}
$$

where $\beta=(\pi / \lambda)\left|\Delta n_{\max }\right| ; \mathrm{d} U_{y}$ is negative because the refractive-index modulation along the $y$ axis is taken to be negative. The elementary diffracted amplitude polarized parallel to the pump-beam polarization is written with the help of Eq. (4):

$$
\mathrm{d} S_{\|}(\text {diffraction })=2 S_{\|} \beta \mathrm{d} z \cos (2 \theta+2 \rho z) .
$$

The change in $S_{\|}$due to absorption in a thickness dz of the crystal is

$$
\mathrm{d} S_{\|}(\text {absorption })=S_{\|}[\exp (-\alpha \mathrm{d} z / 2)-1]=-S_{\|} \alpha \mathrm{d} z / 2,
$$

where $\alpha$ is the absorption coefficient of the crystal. Hence the total change in $S_{\|}$in traversing the crystal thickness $\mathrm{d} z$ is

$$
\mathrm{d} S_{\|}=S_{\|} \mathrm{d} z[2 \beta \cos (2 \theta+2 \rho z)-\alpha / 2] .
$$

The change in $R$ due to absorption is

$$
\mathrm{d} R / \mathrm{d} z=\mathrm{d}\left\{R_{0} \exp [-\alpha(L-z) / 2]\right\} / \mathrm{d} z=\alpha R / 2
$$

or

$$
\mathrm{d} R=\alpha R \mathrm{~d} z / 2,
$$

where $R_{0}$ is the amplitude of the pump beam when it enters the crystal at $z=L$. Substituting Eqs. (7) and (8) into Eq. (2), we obtain

$$
\mathrm{d} m=m \mathrm{~d} z[2 \beta \cos (2 \theta+2 \rho z)-\alpha] .
$$

Integrating Eq. (9) with the initial condition, at $z=0$, $m=m_{0}$, where $m_{0}=\left(2 S_{0} / R_{0}\right) \exp (\alpha L / 2)$, we obtain

$$
m=m_{0} \exp [-\alpha z+(2 \beta / \rho) \cos (2 \theta+\rho z) \sin \rho z] .
$$

\section{B. Calculating the Total Diffracted Amplitude Emerging from the Crystal}

Substituting Eq. (10) into Eq. (4), we obtain the elementary amplitude components diffracted by the grating of thickness $\mathrm{d} z$

$$
\begin{aligned}
\mathrm{d} U_{x}(z)= & R_{0} \exp [-\alpha(L-z) / 2] \cos (\theta+\rho z) \beta m \mathrm{~d} z \\
= & 2 S_{0} \beta \cos (\theta+\rho z) \exp [-\alpha z / 2+(2 \beta / \rho) \\
& \times \cos (2 \theta+\rho z) \sin \rho z] \mathrm{d} z \\
\mathrm{~d} U_{y}(z)= & -2 S_{0} \beta \sin (\theta+\rho z) \exp [-\alpha z / 2+(2 \beta / \rho) \\
& \times \cos (2 \theta+\rho z) \sin \rho z] \mathrm{d} z .
\end{aligned}
$$

These diffracted components propagate in the remaining thickness $(L-z)$ of the crystal; they rotate owing to the optical activity and are partially absorbed. At the exit face these components are again decomposed along the $x, y$ axes. We thus write the diffracted amplitude at the exit plane contributed by the elementary grating situated at $z$ :

$$
\begin{aligned}
\mathrm{d} U_{x}(L)= & \exp [-\alpha(L-z) / 2]\left\{\mathrm{d} U_{x}(z) \cos \rho(L-z)\right. \\
& \left.+\mathrm{d} U_{y}(z) \cos [\rho(L-z)+(\pi / 2)]\right\} \\
= & 2 S_{0} \beta \cos (\theta-\rho L+2 \rho z) \mathrm{d} z \exp [-\alpha L / 2 \\
& +(2 \beta / \rho) \cos (2 \theta+\rho z) \sin \rho z], \\
\mathrm{d} U_{y}(L)= & -2 S_{0} \beta \sin (\theta-\rho L+2 \rho z) \mathrm{d} z \exp [-\alpha L / 2 \\
& +(2 \beta / \rho) \cos (2 \theta+\rho z) \sin \rho z] .
\end{aligned}
$$

The total diffracted-amplitude components $U_{x}$ and $U_{y}$ are calculated by numerically integrating these expressions. The total output signal is the initial signal plus the diffracted amplitude

$$
\begin{aligned}
& S_{x}(z=L)=S_{0} \exp (-\alpha L / 2) \cos (\theta+\rho L)+U_{x}, \\
& S_{y}(z=L)=S_{0} \exp (-\alpha L / 2) \sin (\theta+\rho L)+U_{y} .
\end{aligned}
$$

The signal gain $\gamma$ is calculated as follows: $\quad \gamma$ is the output signal intensity in presence of the pump beam/Output signal intensity without the pump beam,

$$
\gamma=\left(S_{x}{ }^{2}+S_{y}{ }^{2}\right) / S_{0}^{2} \exp (-\alpha L) .
$$

With the assumptions that we made, the output signal is linearly polarized and the direction of polarization is calculated with the ratio $S_{y} / S_{x}$. We note from Eqs. (12)(14) that if $\theta$ is changed to $\theta+\pi$ the expressions for $S_{x}$ and $S_{y}$ change sign, but $\gamma$ remains unchanged. We also note that $\gamma(\theta, \rho)=\gamma(-\theta,-\rho)$. The integral of the expressions in Eq. (12) can be carried out analytically if $\beta / \rho \leqslant 1$; the exponential factor is then expanded in a Taylor's series and a few terms are retained. This inequality is amply satisfied for BSO crystals and to a lesser extent for BTO. We carry out these calculations in Appendix A and give analytic expressions for the signal gain and for the polarization direction of the amplified signal. The results obtained analytically and numerically for the BSO crystal agree to better than $1 \%$.

The results [Eqs. (12)-(14)] derived here for the contradirectional beam coupling apply perfectly to the codirectional coupling when optical activity and crystal absorp- 
tion are taken into account and pump depletion due to transfer of energy is neglected. In the codirectional geometry, known as the transverse geometry because the space-charge field is orthogonal to the general direction of light propagation, the crystal wafer is cut normal to the $\langle 110\rangle$ axis, the axes $\langle 110\rangle$ and $\langle 001\rangle$ lie in the plane of the wafer, and the grating vector is parallel to the $\langle 110\rangle$ axis. In both these configurations we have two linear birefringent axes with refractive indices $n_{0} \pm(1 / 2) n_{0}{ }^{3} r E_{s c}$, the value of $E_{s c}$ is, of course, different in the two cases.

\section{EXPERIMENTAL VERIFICATION OF THE THEORETICAL MODEL}

\section{A. Signal Gain as a Function of $\theta$ : BSO}

The signal gain $\gamma$ and the polarization direction of the amplified signal are measured for a BSO:Mn and a BTO:Cd crystal with a He-Ne laser $(\lambda=633 \mathrm{~nm})$. The ratio $S_{0}{ }^{2} / R_{0}{ }^{2}$ is $7 \times 10^{-3}$. The power density incident upon the crystal is approximately $80 \mathrm{~mW} \mathrm{~cm}^{-2}$. The curve in Fig. 2 represents the signal gain $\gamma$ as a function of the polarization direction $\theta$ of the incident signal beam for the BSO crystal $\left(\rho=-0.39 \mathrm{~mm}^{-1}\right.$, thickness $\left.2.07 \mathrm{~mm}\right)$. The pump-beam polarization is at $\theta+\rho L$. The circles represent the experimentally measured values. The best fit between theoretical and experimental results is obtained for $\beta=0.11 \mathrm{~mm}^{-1}$; this gives the saturation value of the space-charge field to be $7 \mathrm{kV} \mathrm{cm}^{-1}$ ( $r$ is taken to be $4 \times 10^{-12} \mathrm{mV}^{-1}$ ). It should be noted that $7 \mathrm{kV} \mathrm{cm}^{-1}$ is the maximum value of the space-charge field that is obtained for $m=1$; due to the low value of $m$, the actual

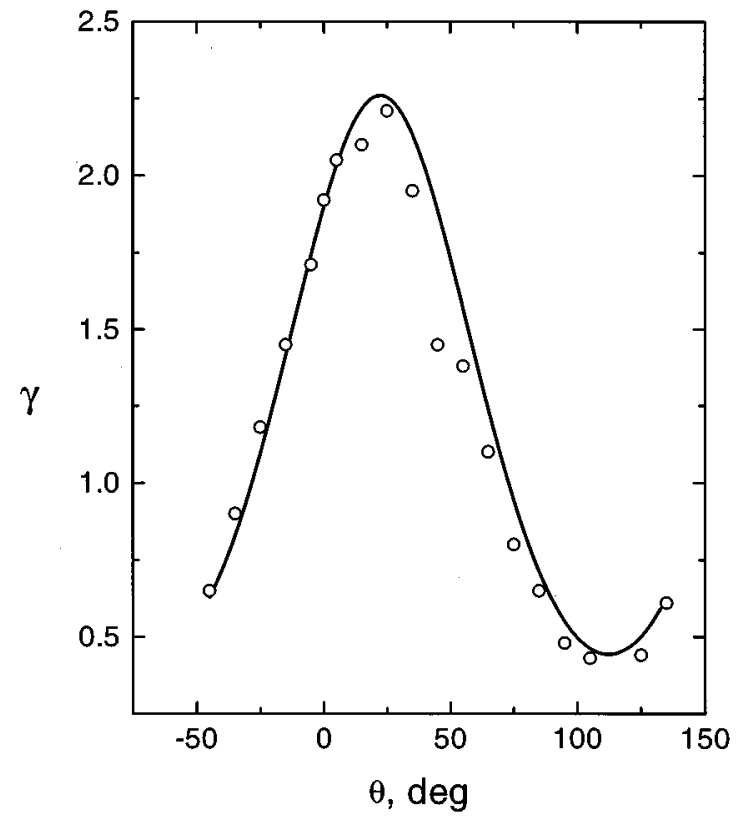

Fig. 2. Variation of the signal gain $\gamma$ as a function of the polarization direction $\theta$ of the incident signal beam for BSO crystal $\left(\rho=-0.39 \mathrm{~mm}^{-1}\right.$; thickness, $\left.2.07 \mathrm{~mm}\right)$. The polarization direction of the pump as it enters the crystal at $z=L$ is $\theta+\rho L$. The solid curve is the theoretical result obtained by the numerical integration of Eq. (12) and by use of Eqs. (13) and (14). The circles represent experimental values. The best fit between theoretical and experimental results is obtained for $\beta$ $=0.11 \mathrm{~mm}^{-1}$. value of $E_{\text {sc }}$ in the crystal is much lower. The theoretical curve in Fig. 2 has been calculated by numerically integrating Eq. 12. However, because $\beta / \rho$ in our case is much less than 1 ( $\beta / \rho=0.11 / 0.39)$, the analytic expression derived in Appendix A gives equally accurate results. In addition, we can draw some interesting conclusions about the signal gain from this expression. Because $C(=\beta / \rho)$ $\ll 1$, we can neglect the third and the higher-order terms in $C$ and write Eq. (A5) in the form

$$
\begin{aligned}
\gamma=1 & +4(\beta / \rho) A \sin \rho L \cos (2 \theta+\rho L) \\
& \times[1+(\beta / \rho) \cos \rho L \sin (2 \theta+\rho L)] \\
& +4(\beta / \rho)^{2} A^{2} \sin ^{2}(\rho L), \\
A= & \exp [-(\beta / \rho) \sin (2 \theta)] .
\end{aligned}
$$

This simplified equation permits us to determine the optimal orientation $\theta$ that maximizes $\gamma$. To study the variation of $\gamma$ as a function of $\theta$, we can ignore the second term within the square brackets in Eq. (15) because it is much smaller than the first term, which is 1 . The parameter $A$ does not vary much with $\theta$ and remains close to unity. The only term that varies significantly with $\theta$ and even changes its sign is

$$
4(\beta / \rho) A \sin \rho L \cos (2 \theta+\rho L) .
$$

For $0<|\rho L|<\pi$, $(\sin \rho L) / \rho$ is positive irrespective of the sign of $\rho$, and this term reaches its maximum value for $\theta=-\rho L / 2$. For $\pi<|\rho L|<2 \pi$, which is true for very thick crystals, the optimal value of $\theta$ is $-\rho L / 2 \pm \pi / 2$. It should be remarked that these optimal values of $\theta$ do not maximize $A$ but, at least, make it greater than 1 and thus result in further enhancement of the signal gain. These observations about the optimal orientation $\theta$ are verified by the experimental and the numerically calculated results presented in Fig. 2. The maximum occurs at $\theta$ $=21^{\circ}$ and the $-\rho L / 2$ value of our BSO crystal is $23^{\circ}$ (our BSO and BTO crystals have a negative rotatory power).

Substituting $\theta=-\rho L / 2$ in Eq. (15), we obtain the maximum value of the signal gain

$$
\gamma_{\max }=\{1+(2 \beta / \rho) \sin \rho L \exp [(\beta / \rho) \sin \rho L]\}^{2} .
$$

Using the values of $\beta, \rho$, and $L$ of our BSO crystal in this equation, we find that $\gamma_{\max }=2.25$. This value agrees to within $1 \%$ with the value obtained by numerically integrating expression (12). This validates our approximation used for writing the simplified expressions (15) for the signal gain, at least so far as $\gamma_{\max }$ is concerned.

In Fig. 3 we draw a few $\gamma-\theta$ curves for different values of $\rho L$ obtained by numerical integration of Eq. (12) and by use of Eqs. (13) and (14). These curves again verify our conclusions about the optimal value of $\theta$. (The curves for which $\rho L$ is an integral multiple of $\pi$ will be discussed separately.) This optimization of the signal- and pumpbeam polarizations was pointed out for the two-beam interaction in transmission geometry by Petrov et al. ${ }^{19}$ and other authors. ${ }^{13}$ In fact the explanation is quite simple: With this choice of the pump and signal polarizations, the polarization vector of the pump and that of the original signal at the middle of the crystal $(z=L / 2)$ are along the $x$ axis (the axis with positive refractive-index modulation), and hence the diffracted amplitude contributed by 


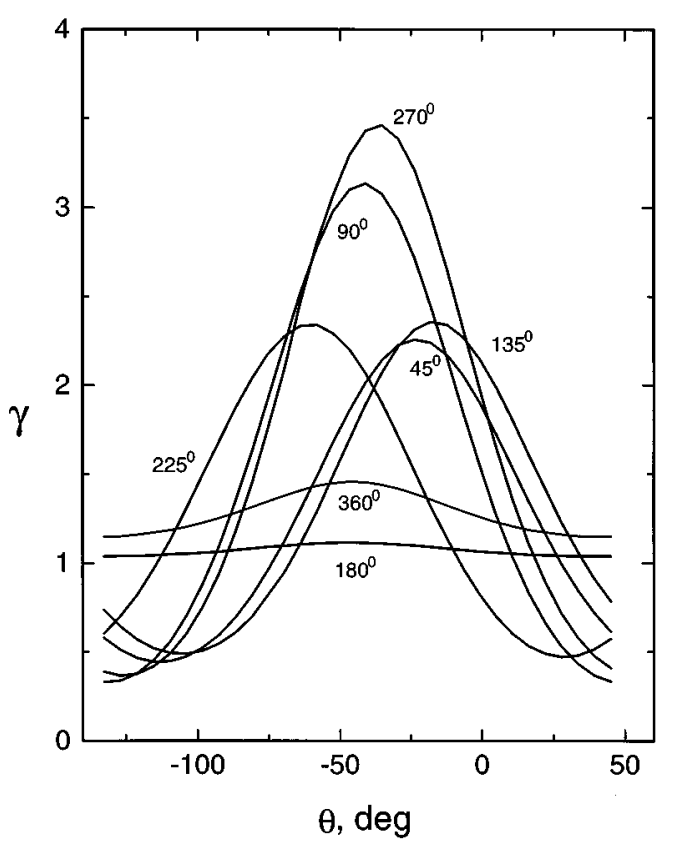

Fig. 3. Numerically calculated $\gamma-\theta$ curves for a BSO crystal $\left(\rho=0.39 \mathrm{~mm}^{-1}, \beta=0.11 \mathrm{~mm}^{-1}\right.$ ) for different values of $\rho L$ $\left(45^{\circ}, 90^{\circ}, 135^{\circ}, 180^{\circ}, 225^{\circ}, 270^{\circ}\right.$, and $\left.360^{\circ}\right)$.

the pump in this region adds in phase to the signal. In other words, the $y$ component of the diffracted amplitude is zero. Besides, if $\rho L \leqslant \pi / 2$, the light vectors stay close to the $x$ axis throughout their transit in the crystal, and thus the amplification is maximum.

1. Signal Gain for $\rho L=k \pi, k$ Being an Integer

Substituting $\rho L=k \pi$ in the analytic expression (A5), we obtain

$$
\gamma=1+(\beta / \rho)^{4} \exp [-(2 \beta / \rho) \sin 2 \theta] k^{2} \pi^{2} .
$$

In practical situations $k$ is rarely greater than 1 , and thus $\gamma$ is close to 1 (no amplification). The maximum occurs for $\theta=45^{\circ}$ (for $\rho$ negative) and $\theta=-45^{\circ}$ (for $\rho$ positive). These conclusions drawn from analytic expressions are verified by the numerically calculated curves for $\rho L=\pi$ and $\rho L=2 \pi$ in Fig. 3. An intuitive explanation of this result is as follows: for $\rho L=\pi$ (or $k \pi$ ), during their transit in the crystal, the pump and the signal beam vectors stay close to the $x$ axis as long as they stay to the $y$ axis. Consequently the amplification (during the passage close to the $x$ axis) and the deamplification (passage close to the $y$ axis) practically cancel each other. This discussion remains valid when $\rho L$ is not exactly $k \pi$ but is close to this value.

\section{Variation of $\gamma$ as a Function of Crystal Thickness}

We observe, from the approximate relations (15)-(16) obtained by neglecting higher than second-order terms in $(\beta / \rho)$, that $\gamma$ will reach its maximum value when $|\rho L|$ $=\pi / 2$. Of course there are some higher-order terms in Eq. (A5), such as $A^{2}(\beta / \rho)^{4}(\rho L)^{2}$, that increase with $L$, but their contribution to $\gamma$ is negligible. Thus there is no point in using a thick crystal with $|\rho L|>\pi / 2$. An intuitive explanation is as follows: With $\rho L=\pi / 2$ and the choice $\theta=-45^{\circ}$, the pump and the signal wave vectors stay close to the $x$ axis throughout their transit in the crystal. If $\rho L>\pi / 2$, the light vectors will be relatively closer to the deamplifying $y$ axis at least during a fraction of their transit. The exact numerical results represented by the curves in Fig. 3 show this effect. The gain increases when $\rho L$ is increased from $45^{\circ}$ to $90^{\circ}$. However, any further gain remains insignificant even when the crystal thickness is increased threefold $\left(\rho L=270^{\circ}\right)$. The same effect is shown by the curves for $\rho L=45^{\circ}$ and $\rho L=225^{\circ}$.

B. $\quad \gamma$ as a Function of $\theta$ and of $L$ : BTO

The curve in Fig. 4 represents $\gamma$ as a function of $\theta$ for a BTO:Cd crystal $\left(\rho=-0.11 \mathrm{~mm}^{-1}, L=4.2 \mathrm{~mm}\right)$ obtained by numerical integration of Eq. (12) and by use of Eqs. (13) and (14). The circles are the experimental results. The best fit between experimental and theoretical results is obtained for $\beta=0.133 \mathrm{~mm}^{-1}$. The maximum of the signal gain occurs for $\theta=12^{\circ}$, which is in excellent agreement with the $\theta=-\rho L / 2$ value for our crystal $(\rho L$ $\left.=-26^{\circ}\right)$. Though the approximation used for writing the simplified expression (15) is not valid for the BTO crystal, the fact remains that the choice of $\theta=-\rho L / 2$ maximizes the signal gain because the interacting beam vectors stay close to the amplifying axis.

For our BTO crystal the value of $\rho L$, the total angle through which the wave vectors rotate during their transit in the crystal, is only $26^{\circ}$. Besides, if we polarize the signal and pump beams as they enter the crystal on opposite faces, at $-\rho L / 2$ and $-\rho L / 2+\rho L$, respectively, the two vectors stay practically parallel to the $x$ axis. Under these conditions (low value of $\rho L$ and appropriately chosen polarizations) the signal gain can be calculated with a

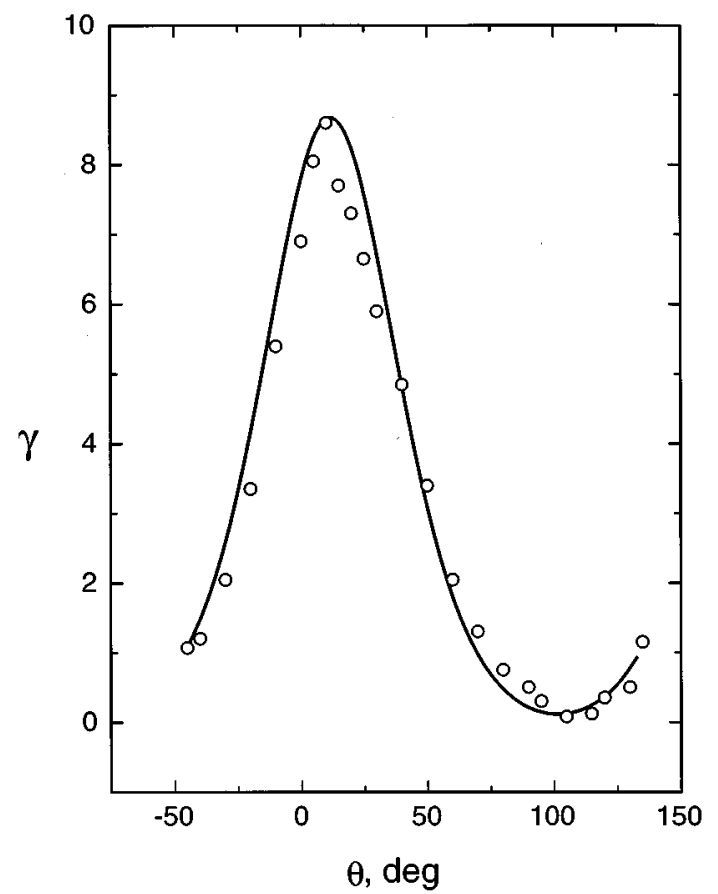

Fig. 4. $\gamma-\theta$ curve for the BTO crystal $\left(\rho=-0.11 \mathrm{~mm}^{-1}, L\right.$ $=4.2 \mathrm{~mm}$ ). The solid curve represents the numerically calculated theoretical result; the circles indicate experimentally measured values. The best fit between theoretical and experimental results is obtained for $\beta=0.133 \mathrm{~mm}^{-1}$. 
good accuracy by taking $\rho=0$ in our analysis. Equation (12) for the elementary diffracted amplitude then becomes

$$
\begin{aligned}
& \mathrm{d} U_{x}(L)=2 S_{0} \beta \cos \theta \exp [-\alpha L / 2+2 \beta z \cos 2 \theta], \\
& \mathrm{d} U_{y}(L)=-2 S_{0} \beta \sin \theta \exp [-\alpha L / 2+2 \beta z \cos 2 \theta] .
\end{aligned}
$$

Integrating these expressions and using Eqs. (13) and (14), we obtain the signal gain

$$
\begin{aligned}
\gamma=1 & +\left(1 / \cos ^{2} 2 \theta\right)[\exp (2 \beta L \cos 2 \theta)-1]^{2} \\
& +2[\exp (2 \beta L \cos 2 \theta)-1] .
\end{aligned}
$$

For optimal gain we take $\theta=0$, that is, we take the beam polarizations parallel to the $x$ axis. This gives

$$
\gamma_{\max }=\exp (4 \beta L) .
$$

If $\theta$ is taken to be $\pi / 2, \gamma$ is equal to $\exp (-4 \beta L)$. Expression (20) is identical to the one obtained in the transmission geometry. Substituting $\beta=0.133 \mathrm{~mm}^{-1}$ and $L$ $=4.2 \mathrm{~mm}$ into Eq. (20), we obtain $\gamma_{\max }=9.3$. The value obtained from the rigorous treatment (Fig. 4) is 8.7. The agreement is highly satisfactory. As expected, the presence of optical activity tends to decrease the gain. For our BSO crystal $\left(\rho L=46^{\circ}\right)$, Eq. (20) gives $\gamma=2.5$, a satisfactory approximation of the exact value 2.25. For applications needing amplification of signal, the BTO crystal has the advantage over BSO in that it has low rotatory power and a relatively higher electro-optic coefficient. A $1-\mathrm{cm}$ thick crystal $\left(\rho L=63^{\circ}\right)$ will give a signal gain of about 100 . Taking into account crystal absorption $\left(\alpha=1 \mathrm{~cm}^{-1}\right)$, the ratio of the output to the input signal intensity $\left(S^{2} / S_{0}{ }^{2}=\gamma \exp (-\alpha L)\right)$ is $\sim 35$. Optimizing the grating period should yield still higher amplifications. It should be remembered that these high gains are obtained without applying any external electric field to the crystal.

\section{Polarization Direction of the Amplified Signal}

The polarization direction $\psi$ of the amplified signal beam is given as a function of $\theta$ in Fig. 5. The angle $\phi$ plotted here is in fact $\phi=\psi-(\theta+\rho L)$. In other words, $\phi$ is the (polarization direction of the output signal in presence of the pump) minus the (polarization direction of the output signal without the pump). For simplifying the experimental operations the pump polarization was set at $\theta$ and not at $\theta+\rho L$. The theoretical result for this configuration is obtained by multiplying the right-hand side of Eq. (12) times $\cos \rho L$ and replacing $\theta$ with $\theta-\rho L$. We again find that the agreement between theoretical and experimental results is excellent.

In Fig. 6 we present $\phi-\theta$ curves for the optimal situation in which the pump-beam polarization is set at $\theta$ $+\rho L$. We note that for $\theta=-\rho L / 2, \phi$ is nearly zero; that is, the polarization of the amplified signal is roughly the same as that of the unamplified signal. Intuitively this can be explained as follows: For our crystals $\rho L<1$ and by choosing the signal and pump polarizations at $-1 / 2 \rho L$ and $+1 / 2 \rho L$, respectively, we ensure that the two vectors stay close to the amplifying $x$ axis throughout their transit. The diffracted light generated by an elementary grating is polarized parallel to the $x$

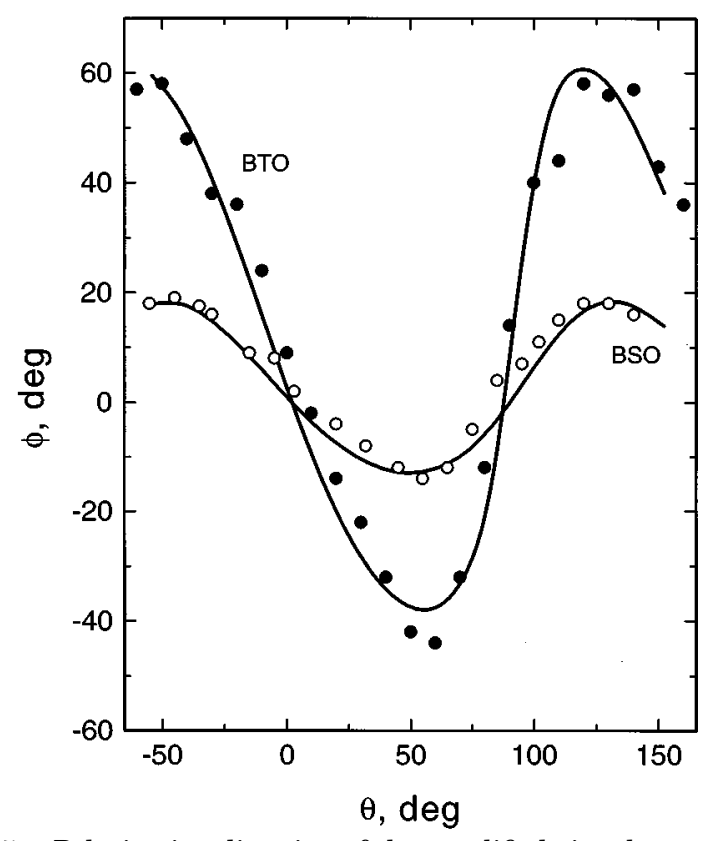

Fig. 5. Polarization direction of the amplified signal as a function of $\theta$. Here $\phi$ is (the polarization direction of the signal at $z=L$ in presence of the pump beam) minus (the polarization direction of the signal at $z=L$ without the pump). The experimental results (circles) are in excellent agreement with the numerically calculated results (solid curves). The theoretical and experimental results in this figure are for the situation in which the pump-beam polarization at $z=L$ is set at $\theta$ and not at $\theta$ $+\rho L$.

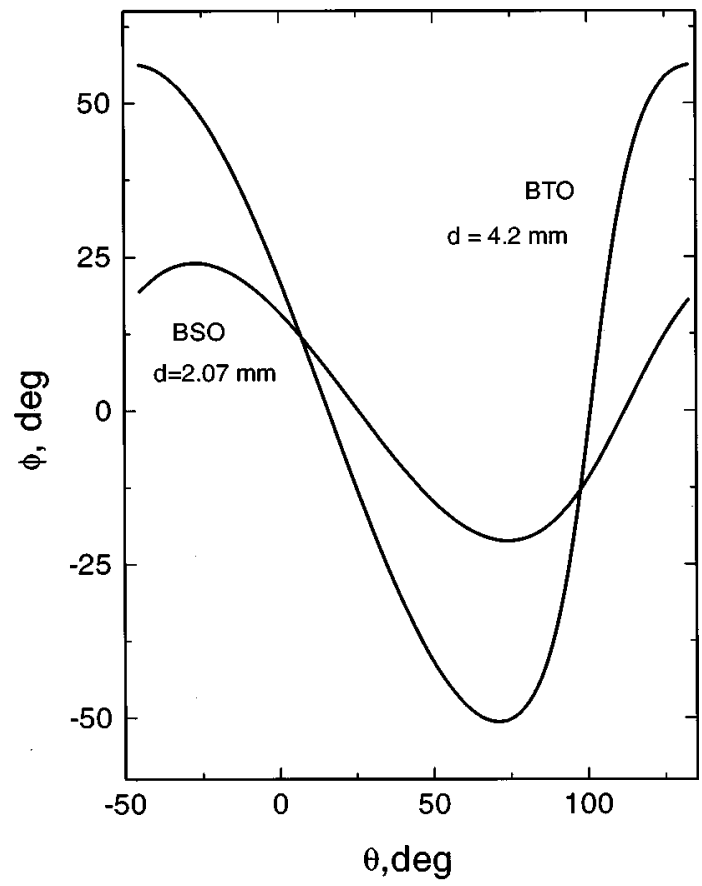

Fig. 6. $\phi-\theta$ curves for the optimal situation in which the pump-beam polarization is set at $\theta+\rho L$.

axis irrespective of the $z$ coordinate of the grating; of course the light vector rotates as it propagates in the remaining thickness $L-z$ of the crystal. Thus the elementary amplitude component generated by an elementary grating located at the middle of the crystal $(z$ 
$=L / 2)$ emerges with its vector at $1 / 2 \rho L$ with respect to the $x$ axis. The amplitude components generated in the first half of the crystal emerge at angles $>1 / 2 \rho L$, and those generated in the second half emerge at angles $<1 / 2 \rho L$. Though these elementary components do not have the same moduli, their resultant vector is approximately at an angle $1 / 2 \rho L$ with the $x$ axis. The signal, in the absence of the pump, also emerges at this angle. Hence the angle $\phi$ is close to zero. We also note that the variations of $\phi$ are much larger for BTO than for BSO. This is so because the contribution of the diffracted light, which determines $\phi$ to a large extent, to the outcoming signal is much higher in the case of BTO.

Our experimental observations show that the amplified signal wave is slightly elliptic; the angle $\psi$ represents the orientation of the major axis of the ellipse. For BSO the ratio of the intensity along the minor axis to that along the major axis is only $1 / 40$, and for BTO it is $1 / 8$. This ellipticity is probably due to the fact that the reflection grating is not shifted by exactly $\pi / 2$ with respect to the interference pattern.

\section{CONCLUSION}

In summary, we have calculated the signal amplification $\gamma$ and its polarization direction in contradirectional twobeam coupling in sillenite crystals. Crystal absorption and its optical activity are taken into account, but the pump depletion due to energy transfer is neglected. The crystal slice is cut normal to one of its crystallographic axes, and the space-charge field is parallel to this axis (say, the $z$ axis). No bias electric field is applied to the crystal. The birefringent axes, $x$ and $y$, induced by the space-charge field have the refractive indices $n_{0}$ $\pm n_{0}{ }^{3} r E_{s c} / 2$, respectively. The signal is amplified when the polarization vectors of the signal and the pump beams stay close to, say, the $x$ axis during their transit in the crystal, and is deamplified when the vectors are close to the $y$ axis. Numerical integration is, in general, necessary to calculate the signal gain. However, an analytic expression for $\gamma$ is obtained for the case $\beta / \rho \leqslant 1(\beta$ $\left.=\pi \Delta n_{\max } / \lambda\right)$. This condition is satisfied by BSO and BGO crystals. This expression shows that the gain is maximum when the polarization vectors of the forwardand the backward-propagating beams are set at $-\rho L / 2$ and $+\rho L / 2$, respectively, and when $|\rho L|=\pi / 2$. With this initial disposition the polarization vectors of the two beams stay close to the amplifying $x$ axis throughout their transit in the crystal, and consequently the signal gain is maximum.

For our BTO crystal, $\beta / \rho=1.2$, and the analytic expression calculated for crystals with relatively high optical activity gives only a rough value that is lower than the exact numerically calculated value. For a BTO crystal of moderate thickness, say, $5-6 \mathrm{~mm}$, the value of $\rho L$ is much less than 1 and an approximate value of the signal gain can be obtained from the well-known expression $\exp (4 \beta L)$ that applies to crystals with no optical activity. Obviously $\exp (4 \beta L)$ gives a value that is higher than the exact value because the presence of optical activity tends to decrease the gain.
The signal gain is maximum when the initial polarization directions of the beams are set at $\pm \rho L / 2$; moreover, the amplified signal has approximately the same polarization direction as it has in the absence of the pump. A very interesting result is that the expressions for the signal gain in contradirectional and codirectional geometries are identical. Identical results are also obtained for these geometries $\left(\mathrm{Ja}^{14}\right.$ and $\left.\mathrm{Yeh}^{2}\right)$ when pump depletion is taken into acount but crystal absorption and optical activity are neglected. The BTO crystal provides high gains and may be useful for phase-conjugation experiments.

\section{APPENDIX A: CALCULATING THE SIGNAL GAIN WHEN $\beta / \rho \leqslant 1$}

The elementary, diffracted amplitude in Eq. (12) can be written in the form

$$
\begin{aligned}
\mathrm{d} U_{x}(L)= & 2 S_{0} \beta \cos (\theta-\rho L+2 \rho z) \exp [-\alpha L / 2 \\
& -(\beta / \rho) \sin 2 \theta] \exp [(\beta / \rho) \sin (2 \theta+2 \rho z)] \mathrm{d} z .
\end{aligned}
$$

A similar equation is written for $\mathrm{d} U_{y}(L)$.

If $(\beta / \rho) \leqslant 1$, we can write

$\exp [(\beta / \rho) \sin (2 \theta+2 \rho z)]$

$$
\begin{aligned}
\approx & 1+(\beta / \rho) \sin (2 \theta+2 \rho z)+\left(\beta^{2} / 2 \rho^{2}\right) \\
& \times\{0.5[1-\cos (4 \theta+4 \rho z)]\} . \quad(\mathrm{A} 2)
\end{aligned}
$$

Substituting relation (A2) into Eq. (A1) and integrating over the crystal thickness, we obtain

$$
\begin{aligned}
U_{x}= & 2 S_{0}(\beta / \rho) \exp [-\alpha L / 2-(\beta / \rho) \sin 2 \theta]\left\{\left[1+\left(\beta^{2} / 4 \rho^{2}\right)\right]\right. \\
& \times \sin \varphi \cos \theta+(\beta / 4 \rho)[2 \varphi \sin (\theta+\varphi) \\
& +\sin 2 \varphi \sin (3 \theta+\varphi)]-\left(\beta^{2} / 8 \rho^{2}\right)[\sin \varphi \cos (3 \theta \\
& +2 \varphi)+(1 / 3) \sin 3 \varphi \cos (5 \theta+2 \varphi)]\},
\end{aligned}
$$

where we have written $\rho L=\varphi$.

By following a similar procedure we find the $y$ component of the total diffracted amplitude:

$$
\begin{aligned}
U_{y}= & -2 S_{0}(\beta / \rho) \exp [-\alpha L / 2-(\beta / \rho) \sin 2 \theta] \\
& \times\left\{\left[1+\left(\beta^{2} / 4 \rho^{2}\right)\right]\right. \\
& \times \sin \varphi \sin \theta+(\beta / 4 \rho)[2 \varphi \cos (\theta+\varphi) \\
& -\sin 2 \varphi \cos (3 \theta+\varphi)]-\left(\beta^{2} / 8 \rho^{2}\right)[-\sin \varphi \\
& \times \sin (3 \theta+2 \varphi)+(1 / 3) \sin 3 \varphi \sin (5 \theta+2 \varphi)]\}
\end{aligned}
$$

Substituting Eqs. (A3) and (A4) into Eq. (13) and using Eq. (14), we find the signal gain $\gamma$ : 


$$
\begin{aligned}
\gamma=1 & +4 A^{2} C^{2}\left(1+C^{2} / 4\right)^{2} \sin ^{2} \varphi+A^{2} C^{4}\left(\varphi^{2}\right. \\
& \left.+\left(\sin ^{2} 2 \varphi\right) / 4\right)+\left(A^{2} C^{6} / 16\right)\left[\sin ^{2} \varphi+(1 / 9) \sin ^{2} 3 \varphi\right] \\
& +4 A C\left(1+C^{2} / 4\right) \sin \varphi \cos (2 \theta+\varphi) \\
& +A C^{2} \sin 2 \varphi \sin (4 \theta+2 \varphi)-\left(A C^{3} / 2\right) \\
& \times[\sin \varphi \cos (2 \theta+\varphi)+(1 / 3) \sin 3 \varphi \cos (6 \theta+3 \varphi)] \\
& +2 A^{2} C^{3}\left(1+C^{2} / 4\right) \sin \varphi \sin (2 \theta+\varphi)[2 \varphi \\
& +\sin 2 \varphi]-A^{2} C^{4}\left(1+C^{2} / 4\right) \sin \varphi \cos (4 \theta+2 \varphi) \\
& \times[\sin \varphi+(1 / 3) \sin 3 \varphi]-A^{2} C^{4} \varphi \sin 2 \varphi \cos (4 \theta \\
& +2 \varphi)+\left(A^{2} C^{5} / 2\right) \varphi[\sin \varphi \sin (2 \theta+\varphi) \\
& -(1 / 3) \sin 3 \varphi \sin (6 \theta+3 \varphi)] \\
& +\left(A^{2} C^{5} / 4\right) \sin 2 \varphi[(1 / 3) \sin 3 \varphi \sin (2 \theta+\varphi) \\
& -\sin \varphi \sin (6 \theta+3 \varphi)] \\
& \left.+\left(A^{2} C^{6} / 24\right) \sin \varphi \sin 3 \varphi \cos (8 \theta+4 \varphi)\right],
\end{aligned}
$$

with $C=\beta / \rho ; A=\exp [-(\beta / \rho) \sin 2 \theta]$.

For BSO and BGO crystals $\beta$ is much less than $\rho$, and therefore $C \ll 1(C=0.28$ in our case $)$ and $A$ remains roughly equal to 1 . Under this condition the expression for $\gamma$ can be simplified by retaining of the terms up to the second order in $C$.

\section{REFERENCES}

1. P. Günter and J.-P. Huignard, Photorefractive Materials and their Applications $I$ and II, Vols. 61 and 62 of Topics in Applied Physics (Springer-Verlag, Berlin, 1988, 1989).

2. P. Yeh, "Contradirectional two-wave mixing in photorefractive media," Opt. Commun. 45, 323-326 (1983).

3. M. D. Ewbank, R. A. Vazquez, R. R. Neurgaonkar, and F. Vachss, "Contradirectional two-beam coupling in absorptive photorefractive materials: application to Rh-doped strontium barium niobate," J. Opt. Soc. Am. B 12, 87-98 (1995).

4. D. Erbschloe, L. Solymar, J. Takacs, and T. Wilson, "Twowave mixing in reflection holograms in photorefractive materials,” IEEE J. Quantum Electron. 24, 820-826 (1988).

5. T. Y. Chang and R. W. Hellwarth, "Optical phase conjuga- tion by backscattering in barium titanate," Opt. Lett. 10, 408-410 (1985).

6. G. C. Valley, "Competition between forward and backward stimulated scattering in $\mathrm{BaTiO}_{3}$," J. Opt. Soc. Am. B 4, 14-19 (1987).

7. A. V. Mamaev and V. V. Shkunov, "Interaction of counterpropagating waves and phase self-conjugation in a $\mathrm{BaTiO}_{3}$ crystal," Sov. J. Quantum Electron. 19, 1199-1203 (1989).

8. A. Marrakchi, R. V. Johnson, and A. R. Tanguay, Jr., "Polarization properties of photorefractive diffraction in electrooptic and optically active sillenite crystals (Bragg regime)," J. Opt. Soc. Am. B 3, 321-336 (1986).

9. S. Mallick, D. Rouède, and A. G. Apostolidis, "Efficiency and polarization characteristics of photorefractive diffraction in a $\mathrm{Bi}_{12} \mathrm{SiO}_{20}$ crystal," J. Opt. Soc. Am. B 4, 1247-1259 (1987).

10. V. V. Shepelevich, S. M. Shandarov, and A. E. Mandel, "Light diffraction by holographic gratings in optically active photorefractive piezocrystals," Ferroelectrics 110, 235-249 (1990).

11. J. R. Goff, "Polarization properties of transmission and diffraction in BSO-a unified analysis," J. Opt. Soc. Am. B 12, 99-116 1995

12. A. Marrakchi, R. V. Johnson, and A. R. Tanguay, Jr., "Polarization properties of enhanced self-diffraction in sillenite crystals," IEEE J. Quantum Electron. QE-23, 2142-2151 (1987).

13. S. Mallick and D. Rouède, "Influence of the polarization direction on two-beam coupling in photorefractive $\mathrm{Bi}_{12} \mathrm{SiO}_{20}$ : diffusion regime," Appl. Phys. B 43, 239-245 (1987).

14. Y. H. Ja, "Energy transfer between two beams in writing a reflection volume hologram in a dynamic medium," Opt. Quantum Electron. 14, 547-556 (1982).

15. Y. H. Ja, "Beam coupling and decoupling in degenerate twowave mixing in a reflection geometry with photorefractive $\mathrm{Bi}_{12} \mathrm{GeO}_{20}$ crystals," Opt. Quantum Electron. 16, 399-404 (1984).

16. N. Kukhtarev, G. Dovgalenko, and V. Starkov, "Influence of the optical activity on hologram formation in photorefractive crystals," Appl. Phys. A 33, 227-230 (1984).

17. M. Miteva and L. Nikolova, "Polarization-dependent selfinduced changes in the optical rotation and optical transmittance in doped crystals of the sillenite type," J. Mod. Opt. 43, 1801-1809 (1996).

18. N. Kukhtarev, B. Chen, P. Venkateswarlu, G. Slamo, and M. Klein, "Reflection holographic gratings in [111] cut $\mathrm{Bi}_{12} \mathrm{TiO}_{20}$ crystal for real time interferometry," Opt. Commun. 104, 23-28 (1993).

19. M. Petrov, T. Pencheva, and S. Stepanov, "Light diffraction from volume phase holograms in electrooptic photorefractive crystals," J. Opt. (Paris) 12, 287-292 (1981). 